\title{
Le comblement du sinus maxillaire : contre-indications absolues et relatives. Gestion des complications
}

\author{
Jabbour M \\ Service de Stomatologie et Chirurgie maxillo-faciale, CHU de Bicêtre, Paris, France \\ michel.jabbour@wanadoo.fr
}

Le rehaussement du plancher sinusien en vue de la pose d'implants dentaires est maintenant un acte bien codifié.

L'utilisation des matériaux de comblement xénogéniques ou allogéniques ont largement contribué au développement de cette technique et à la rationalisation du geste opératoire.

Néanmoins, le sinus maxillaire est une entité anatomique en relation directe avec le sinus ethmoïdal, le sinus frontal, les cavités nasales, etc. Il présente parfois des anomalies et ou des pathologies qui peuvent constituer des contre-indications absolues (pathologie tumorale, polyposes...) ou relatives (aspergillose, kyste dentaire...).

Le chirurgien implantologiste se doit donc d'être très vigilant avant la réalisation de cet acte en s'entourant d'examens complémentaires (panoramique, CT-scan) pour poser un diagnostic précis et réaliser le traitement adéquat visant à rétablir une anatomie et une physiologie du sinus compatibles avec ce rehaussement.

En cas de doute, le recours à un ORL connaissant bien notre spécialité doit être systématique. 